\title{
The coupled dynamics of two particles with different limit sets
}

\author{
C. Mulhern ${ }^{1,2 *}$, D. Hennig ${ }^{2}$, and A.D. Burbanks ${ }^{2}$ \\ ${ }^{1}$ Max Planck Institute for the Physics of Complex System, 01187 Dresden, Germany and \\ ${ }^{2}$ Department of Mathematics, University of Portsmouth, Portsmouth, PO1 3HF, UK ${ }^{*}$
}

\begin{abstract}
We consider a system of two coupled particles evolving in a periodic and spatially symmetric potential under the influence of external driving and damping. The particles are driven individually in such a way that in the uncoupled regime, one particle evolves on a chaotic attractor, while the other evolves on regular periodic attractors. Notably only the latter supports coherent particle transport. The influence of the coupling between the particles is explored, and in particular how it relates to the emergence of a directed current. We show that increasing the (weak) coupling strength subdues the current in a process, which in phase-space, is related to a merging crisis of attractors forming one large chaotic attractor in phase-space. Further, we demonstrate that complete current suppression coincides with a chaos-hyperchaos transition.
\end{abstract}

\section{INTRODUCTION}

Nonlinear transport processes continue to be of vital importance to the understanding of many physical systems. In particular, the transport of particles in symmetric and periodic potential landscapes has attracted considerable interest [1]-[6]. This ubiquitous class of potentials lends itself to a vast number of applications including Josephson junctions [7], charge density waves, nano engines [8], and transport in biological systems [9]. More recently, a study has examined features of the possible interaction of a Josephson junction with axionic dark matter [10]. They note the similarity between the equations of motion of axions and of Joesphson junctions (indeed the equations of motion used in the present study are strikingly similar to the coupled system consisting of an axion and a Josephson Junction) and highlight that their approach of coupling the two could lead to the future development of axionic dark matter detection.

The two-particle system (dimer) is widely studied in the context of these models precisely because it is the smallest form consisting of coupled units and gives insight into what happens in systems of larger scale. The formation of a bond between particles (monomers), often referred to as dimerisation, plays an important role in transport processes. This bond, on the one hand, can allow particles trapped in a potential well to escape to some attracting domain, which individually they would be unable to do [11]. On the other hand, this bond can cause erratic movement of the particles making up the dimer, and thus exclude the possibility of a coherent escape process. Renewed interest in two-particle systems has also come about due to recent work on Bose-Einstein condensates [12].

Another interesting feature of two-particle systems, as compared to single-particle systems, is that a new type of motion is now possible - namely, hyperchaos [13]. Hyperchaos occurs when more than one of the system's Lyapunov exponents is positive. This type of motion is char-

\footnotetext{
* Corresponding author: mulhern@pks.mpg.de
}

acterised by the chaotic diffusion of trajectories in two (or more) different phase-space directions. Some have related the chaos-hyperchaos transition to a change in the stability of an infinite set of unstable periodic orbits which, when a critical transition point is crossed, act as repellers [14].

In this paper we explore the deterministic nonlinear dynamics of two driven and damped coupled particles evolving in a periodic and symmetric potential landscape. Individually, the dynamics of the particles is such that one particle evolves on a chaotic attractor, while the other evolves on a periodic attractor. The dynamics of a similar system, where the driving force for each particle is equal, has been studied recently [15]. It was shown that coordinated energy exchange between the particles takes place such that they are able to surmount consecutive barriers of the periodic potential resulting in coherent transport. Similarly, in the present study we are interested in the dynamics when the particles are coupled to one another. This coupling induces a competition between two dynamical regimes. Therefore, the strength of the coupling will play a key role. In particular, in the scope of weak coupling we wish to establish a critical coupling strength, beyond which directed particle transport is excluded. In previous studies of one-particle systems, a bifurcation point, triggering a current reversal or a current suppression, has been readily obtained numerically $[16,17]$. However, the higher dimensional nature of the system explored in this paper makes finding such a bifurcation point more difficult. To this end, we calculate the Lyapunov exponents for the system and show that the emergence of a vanishingly small current coincides with the transition of the second largest Lyapunov exponent to zero and subsequently becoming positive. Further, by examining three-dimensional projections of the phase-space we will show that attractors, providing directed transport in opposite directions for low coupling strengths, expand and finally merge as a result of a merging crisis as the coupling strength, playing the role of the bifurcation parameter, is increased. This coalescence of the attractors means that transporting channels become suppressed with the particle dynamics being captured on a single chaotic attractor preventing the occurrence of a 
current at all.

The paper is organised as follows: In the next section we introduce the system and discuss the individual particle dynamics; in section III we statistically assess the mean velocity which we relate to the system's Lyapunov spectrum and then later in section IV to the structures in phase-space; we conclude in section $\mathrm{V}$ with a summary.

\section{SYSTEM}

Let us consider a system of two coupled particles, each evolving in a spatially periodic potential with spatial period $L=1$, subjected to damping and periodic driving. The system is modelled by the following set of coupled ordinary differential equations

$$
\begin{aligned}
& \ddot{q}_{1}=-\sin \left(2 \pi q_{1}\right)-\gamma \dot{q}_{1}+h_{1} \sin \left(\Omega t+\theta_{0}\right)-\kappa\left(q_{1}-q_{2}\right)(1) \\
& \ddot{q}_{2}=-\sin \left(2 \pi q_{2}\right)-\gamma \dot{q}_{2}+h_{2} \sin \left(\Omega t+\theta_{0}\right)+\kappa\left(q_{1}-q_{2}\right)(2)
\end{aligned}
$$

where the parameters $\gamma \geq 0, h_{1,2} \geq 0$, and $\kappa \geq 0$ regulate the strength of damping, driving, and coupling respectively. The driving frequency, common to both particles, is given by the parameter $\Omega \geq 0$. Throughout the paper the phase of the external driving field is $\theta_{0}=0$. Quite often in our study $\kappa$ will be used as a control parameter. Note that the exchange symmetry $\left(\dot{q}_{1}, q_{1}\right) \longleftrightarrow\left(\dot{q}_{2}, q_{2}\right)$ is not present in this model, except when $h_{1}=h_{2}$.

Before exploring the system's coupled dynamics, it is important to consider the individual particle dynamics, i.e., the case where $\kappa=0$. For this study, $h_{1}$ is chosen such that the corresponding particle dynamics are chaotic, while the choice of $h_{2}$ will allow for regular dynamics. To this end, we employ a Poincaré map, taking as stroboscopic time the period of the external driving $T=2 \pi / \Omega$. The results are contained in Fig. 1, where the parameters used are $\gamma=0.1, h_{1}=1.3, h_{2}=1.5$, $\Omega=2.25$, and $\kappa=0$. Two attractors can be seen in the $(p=\dot{q}, q)$-plane. The strange attractor corresponds to a driving strength of $h_{1}=1.3$, while the periodic attractor results from a driving strength of $h_{2}=1.5$. In addition, the figure contains an example trajectory illustrating the systems uncoupled dynamics. The two dynamical regimes are clearly distinguishable.

\section{EMERGENCE OF A CURRENT}

The true complexity of this system is revealed only when the individual units making up the dimer are coupled together, i.e. when $\kappa \neq 0$. To gain a quantitative perspective on how $\kappa$ influences the system dynamics we compute the current $v$. More precisely, we compute the time averaged mean velocity for an ensemble of initial conditions, which is given by

$$
v=\frac{1}{T_{s}} \int_{0}^{T_{s}} d t^{\prime}\left\langle\sum_{i=1}^{2} \dot{q}_{i}\left(t^{\prime}\right)\right\rangle,
$$

with simulation time $T_{s}$ and with $\langle\cdot\rangle$ denoting the ensemble average. These initial conditions are chosen such that in the uncoupled case one particle lies on the chaotic attractor, and the other on the periodic attractor seen in Fig. 1. In more detail, the initial conditions corresponding to the subsystem with a driving force of strength $h_{1}$ have positions $q_{1}(0)$ that are distributed uniformly over the (spatial) period of the potential and randomly chosen velocities $\dot{q}_{1}(0)$ with $\left|\dot{q}_{1}(0)\right| \leq 0.5$. The initial conditions corresponding to the subsystem with driving strength $h_{2}$ were chosen so that the particle undergoes regular rotational motion. That is, they were chosen from the basin of attraction of the forward evolving period attractor.

It is clear that when $\kappa=0$ there will be a directed current supplied by the particle on the periodic attractor. The particle on the chaotic attractor will (if at all), on long time scales, provide a vanishingly small contribution to the current. For the computation of the current in chaotic systems we refer to [18].

The key question then is, what happens to the current when the dynamics is in the coupled regime?

For computation of the long-time average, using an ensemble of $N=5000$ initial conditions, the simulation time interval for each trajectory is taken as $T_{s}=10^{5}$. (The simulation time $T_{s}$ exceeds, by magnitudes of order, the typical time scale of the system given by the period duration of oscillations near the bottom of a potential well amounting to $T_{0}=2 \pi / \omega_{0}=\sqrt{2 \pi}$.) Further, the current was calculated for 1000 (evenly spaced) values of $\kappa$ in the range $0 \leq \kappa \leq 0.04$. The results are contained in the bottom panel of Fig. 2 .

As expected, for $\kappa=0$ there is indeed a directed current. With low values of $\kappa$ a number of current reversals can be observed (shown on bottom inset in Fig. 2). In the window $0.001 \lesssim \kappa \lesssim 0.015$ a negative current is supported, followed by a transition to zero. It is this transition, and the subsequent negligible current which is of most interest to us. To explain this we will look at the Lyapunov spectrum for the system given by the equations of motion Eqs. (1)-(2).

The Lyapunov spectrum was computed using the method of Gram-Schmidt orthonormalisation [19], which allows for the simultaneous estimation of all of the systems Lyapunov exponents. The results are contained in the top panel of Fig. 2. As was mentioned earlier, the passing from an uncoupled to a coupled regime can be seen to mark the beginning of a competition between two dynamical regimes. With very weak coupling it is the regular dynamics that prevail. Not only can a directed current be found, but also the effect of chaos is, to an extent, suppressed. Looking more closely at the only positive Lyapunov exponent in this regime (inset Fig. 2), we see that when the particles become coupled 

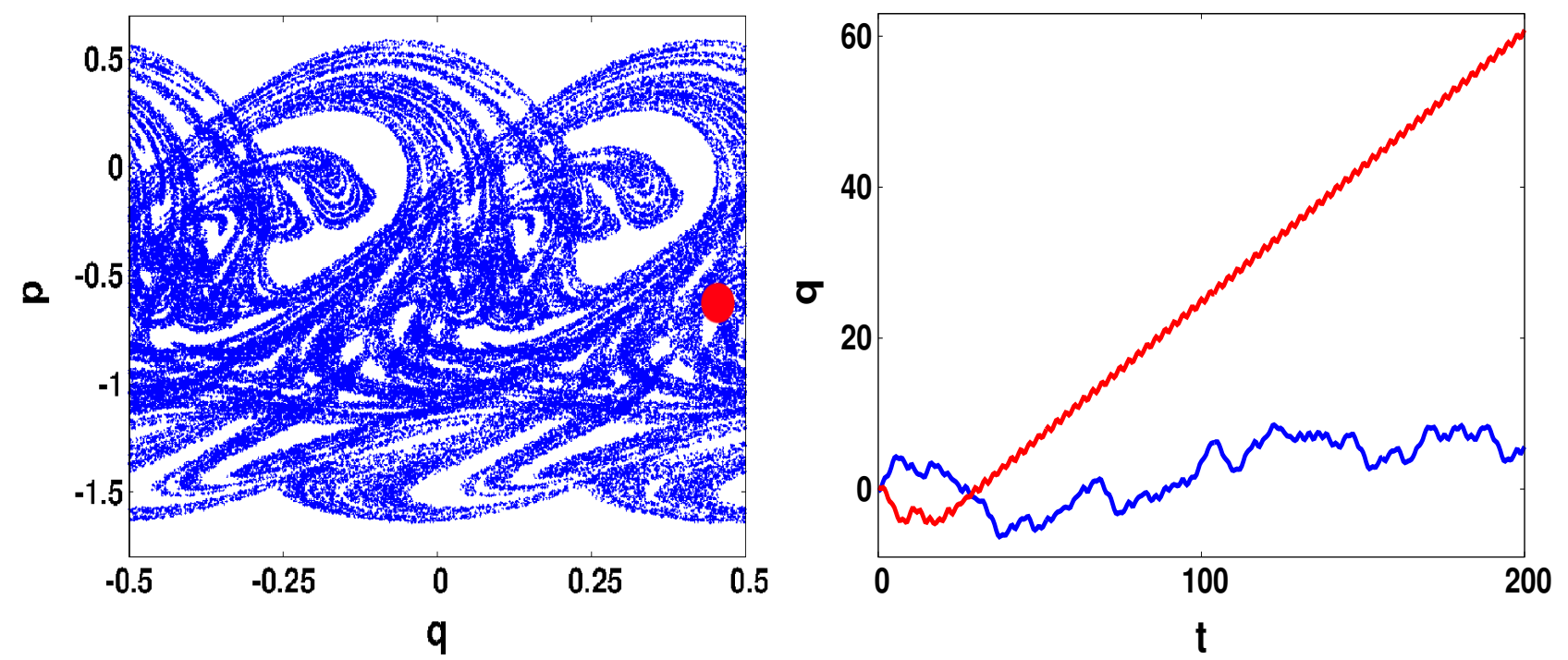

Figure 1. Left panel: Stroboscopic plot showing the strange (blue) and periodic (red) attractors corresponding to driving strengths $h_{1}=1.3$ and $h_{2}=1.5$ respectively, in the uncoupled regime. The remaining system parameters are $\gamma=0.1$, $\Omega=2.25, \theta_{0}=0$, and $\kappa=0.0$. . The dot (red) has been enlarged for emphasis. Right panel: A corresponding example trajectory for motion on the strange (blue line) and periodic (red line) attractor. Note, in the left panel the coordinates $q$ are given $\bmod (1)$.

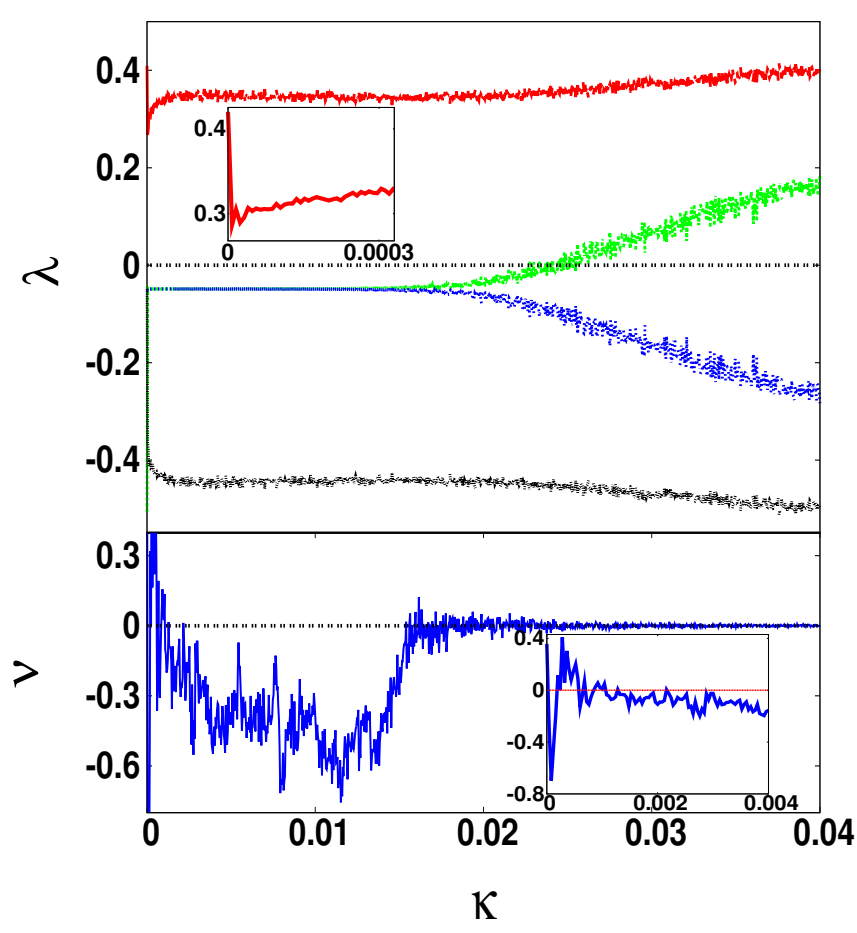

Figure 2. The particle current $\nu$, defined in section III, and the Lyapunov exponents $\lambda$, as a function of the coupling strength $\kappa$. The black (dashed) lines serve to guide the eye. The remaining system parameters are given in Fig. 1. The top and bottom insets show the largest Lyapunov exponent and the current, respectively, for low values of $\kappa$. an instant reduction in the magnitude of the exponent follows. For low values of $\kappa$ the spectrum remains almost constant with one positive and three negative Lyapunov exponents. In the range $0.016 \lesssim \kappa \lesssim 0.024$ two of the exponents diverge from one another. Crucially, this divergence sees one of the exponents approach zero. This divergence also coincides with a reduction in the magnitude of the current (see Fig. 2). At a critical coupling value $\kappa_{c} \approx 0.024$ a second exponent becomes positive marking the transition to hyperchaos. Looking again at Fig. 2, it can be seen that the current is almost negligible. Thus, the transition to hyperchaos has removed any inherent bias in the system and now no direction of motion is favoured, i.e. the possibility of a directed current is excluded. In the next section we will discuss phasespace structures and how they change with increasing $\kappa$, and in addition relate them to the current and Lyapunov spectrum discussed in this section.

Before moving on, it is worth discussing the generality of the results presented in this section. First of all, scanning the entire parameter space is (if possible at all) practically infeasible. Moreover, a change in parameters has implications for the location and types of attractors that can be found in phase-space. However, simulations reveal that the hyperchaos - zero current relation persists when the parameters are chosen from the regular and periodic windows observed in the bifurcation diagram of the single particle. For illustrastion, Fig. 3 shows the current and Lyapunov spectrum, as a function of the coupling strength, for a system that is qualitatively different from that which is the focus of this paper. In more detail, this system has two subsystems were the underlying attractors are both regular. Importantly, the regime of hyper- 


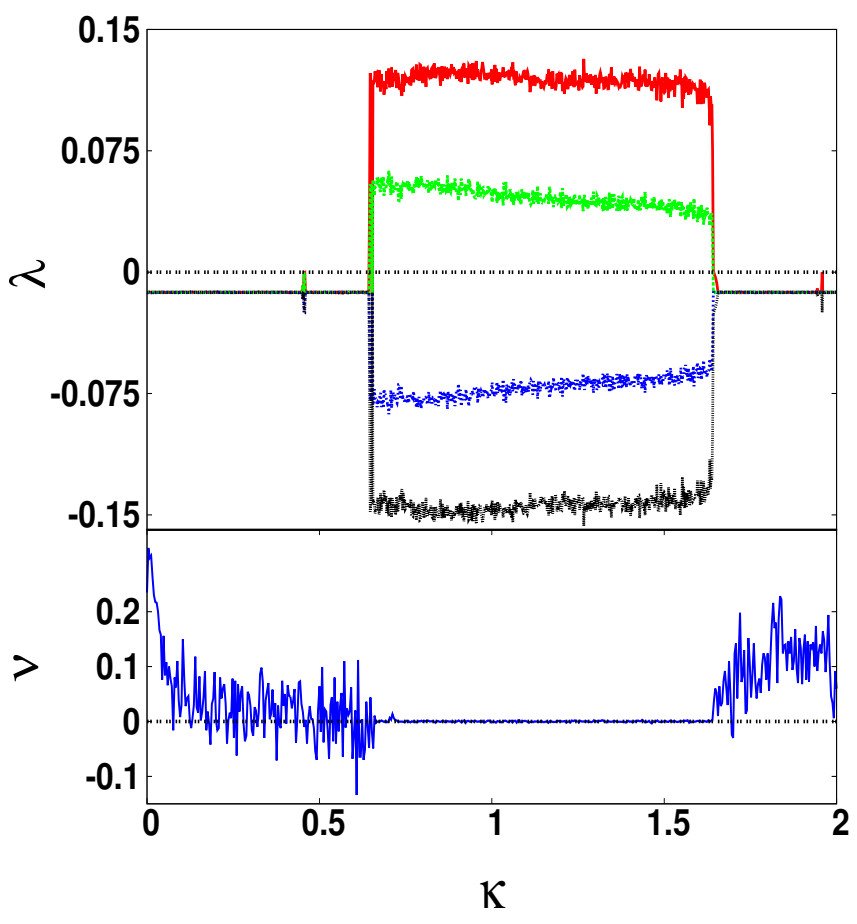

Figure 3. The particle current $\nu$, defined in section III, and the Lyapunov exponents $\lambda$, as a function of the coupling strength $\kappa$. The black (dashed) lines serve to guide the eye. The system parameters are the same as given in Fig. 1, except for $h_{1}=h_{2}=1.5$.

chaos coincides with the window of zero current.

\section{PHASE-SPACE STRUCTURES}

We have seen in the uncoupled regime, i.e. noninteracting particles, that it suffices to examine (stroboscopically) the phase-space for each particle individually (see Fig. 1). However, when the particles are coupled the phase-space becomes five dimensional and such representations, yielding four dimensional Poincaré surfaces of section (PSSs), are no longer suitable. We instead will present three dimensional projections of the four dimensional PSSs illustrating the changes in phase-space through increased coupling. Further, for each $\kappa$ there are four possible three dimensional projections that illustrate qualitatively the same point, and thus we will omit three of them.

Fig. 4(a) shows one of the three dimensional projections for the case of weak coupling, $\kappa=0.01$. It shows two distinct attractors (each having its own basin of attraction which are separated by impermeable basin boundaries) that take the particles in opposite directions. Strikingly, although these are chaotic attractors, indicated by a positive Lyapunov exponent (cf. previous section), the motion on them is nevertheless effectively directed. Like in the uncoupled case, for the system driven at $h_{2}=1.5$, the different weights attached to these transporting attractors favour a directed current (Fig. 2), a feature that is maintained up to coupling strengths $\kappa \lesssim 0.016$. We recall that in a single particle system, i.e. $\kappa=0$, the directed motion results from a lowering of the dynamical symmetry caused by the external modulation field [20],[21]. That is, even though the potential and the external driving field are symmetric with respect to space and time respectively, with the choice of a fixed phase $\theta_{0}$ the symmetry of the flow is reduced and a phase-dependent net motion is found. Due to symmetry reasons it holds that the sign of the mean velocity is reversed upon the changes $\theta_{0}=0 \rightarrow \theta_{0}=\pi$ and $h_{2} \rightarrow-h_{2}$ respectively. However, there exists a phase $0<\theta_{0}<\pi$ for which symmetry between the two coexisting periodic attractors, supporting solutions with velocities of opposite sign, $v_{2}$ and $-v_{2}$, is restored and therefore the net motion vanishes. Hence, in this case for the coupled system the two attractors and their respective basins of attraction are symmetric in phase-space.

As previously mentioned, the interesting feature of Fig. 2 was the transition to, and subsequent negligible current (with increasing $\kappa$ ). When the coupling strength, playing the role of the bifurcation parameter, is increased the three dimensional projections of the PSSs reveal that the two attractors get larger and eventually merge (cf. 4(b)) and 4(c)). That is, a merging crisis takes place for which, at a critical value $\kappa_{m} \simeq 0.015$, the two enlarged attractors collide simultaneously with the basin boundary which separates their basins of attraction. As a result they eventually merge, after the crisis, forming a single large chaotic attractor in phase-space [13],[22]. Note for the coupling strength $\kappa_{m} \simeq 0.015$, when the crisis occurs, there is only one positive Lyapunov exponent. The beginning of the crisis coincides with the event when two of the Lyapunov exponents diverge from each other, with one of them rapidly approaching zero upon increasing the value of $\kappa$ (corresponding to the green and blue line in Fig. 2). At the same time the current rapidly approaches zero. It is also worth noting that if just one of the four three-dimensional projections shows separated attractors, then the attractors are separated in the four-dimensional phase-space. Conversely, to observe a merging of the attractors in the four-dimensional phase-space, it requires that all four three-dimensional projections show a merging of the attractors.

Moreover, for under-critical values of $\kappa_{m} \lesssim \kappa \lesssim \kappa_{c}$, when the degree of merging is not too pronounced (cf. Fig. 4(b)) so that the structure of the formerly separated attractors is still discernible, trajectories jump in a random fashion from the remnant of one of the attractors, after having spent a considerable amount of time there, to the other one and vice versa. As the coupling strength is increased these sojourn times get shorter and shorter. This is illustrated in Fig. 4(b), where, as the result of growth and more pronounced merging, the attractor is covering larger regions of phase-space and the associated net current is already very close to zero. The final three dimensional projection Fig. 4(c) with $\kappa=0.04$ shows the 

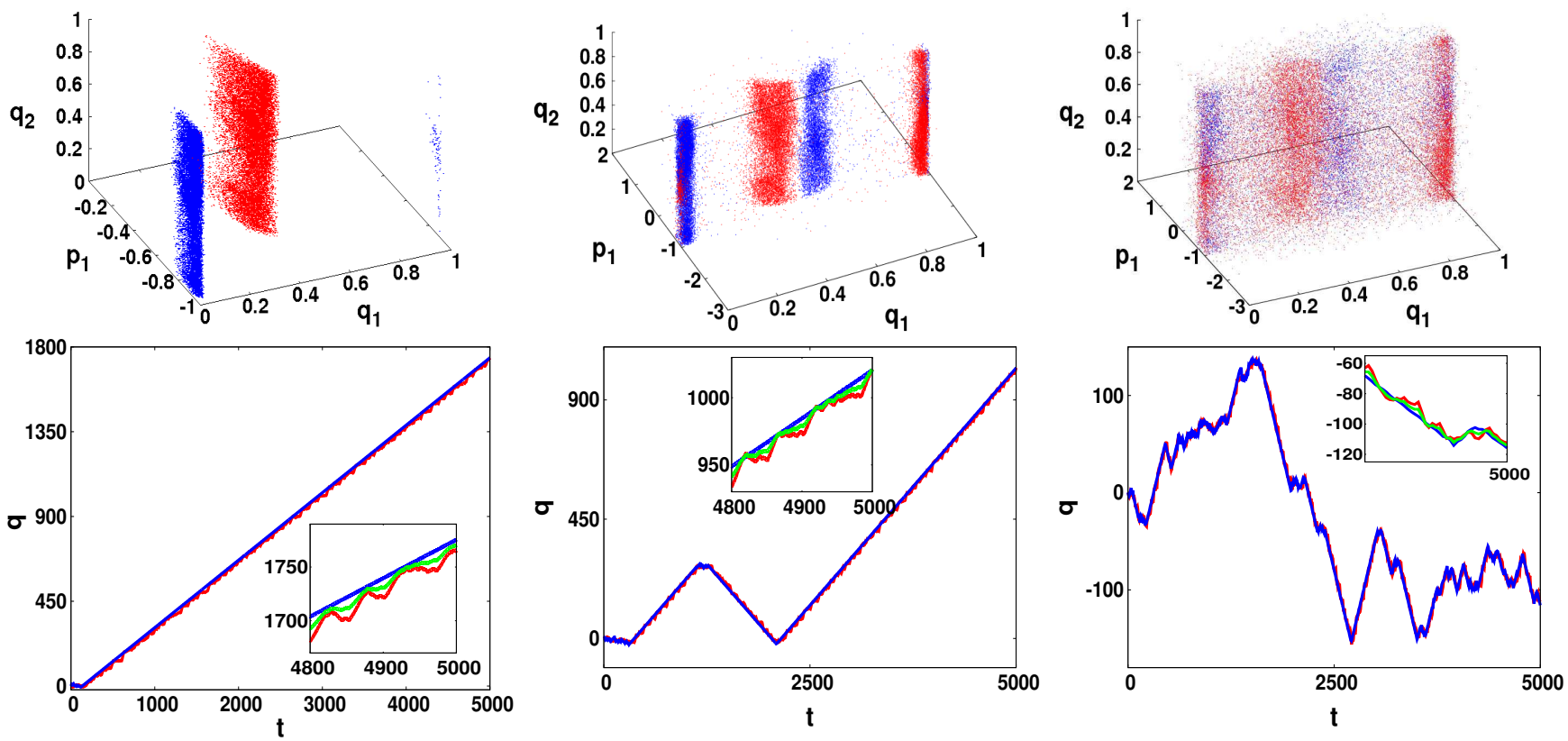

(a)

(b)

(c)

Figure 4. Top row: Three dimensional projections of the attractors in phase-space for different coupling strengths. Left: $\kappa=0.01$, Middle: $\kappa=0.02$, Right: $\kappa=0.04$. In the left panel the attractor shown in blue (red) provides coherent transport to the right (left). In the middle and right panel blue (red) points belong to such orbits whose initial conditions are situated in the basin of attraction of a transport-providing attractor shown in the left panel in blue (red). The remaining parameter values are given in Fig. 1. Bottom row: Examples of corresponding trajectories with the centre of mass shown in green.

phase-space when hyperchaos is well established. In addition, this coupling strength corresponds to a vanishingly small current. The two attractors have now completely merged indicating that no direction of motion is favoured. In other words, symmetry between forward and backward motion is restored. To demonstrate that the attractors have completely merged, we present in Fig. 5 all possible two-dimensional projections of the four-dimensional phase-space.

The features of the attractors before and after the merging crisis are reflected in the time evolution of the trajectories displayed in Fig. 4. In more detail, for coupling strength $\kappa=0.01$ the trajectory in the left panel of Fig. 4 resembles a transporting periodic trajectory of the uncoupled regime. Only a magnification of the plot reveals the chaotic wiggling of the trajectories around the seemingly straight line corresponding to unidirectional particle transport. As the two attractors in phase-space are separated the motion of the particles, associated with a pair of trajectories captured by one of the attractors, proceeds unidirectionally.

In contrast, for coupling strength $\kappa=0.02$, i.e. after the merging crisis, the pair of trajectories shown in Fig. 4 (b) undergoes sudden changes in direction belonging in phase-space to transitions between the 'skeletons' of the two attractors having been transporting before the merging crisis. Finally, in the hyperchaotic regime for $\kappa=0.04$ the pair of trajectories exhibits no coherent properties at all (cf. Fig. 4 (c)). In phase-space there is a single large chaotic attractor left and thus, the 'skeletons' of the formerly transporting attractors have completely disappeared.

\section{CONCLUSION}

We have investigated the dynamics of two coupled driven and damped particles whose individual motions sees one particle evolving on a periodic attractor, and the other evolving on a strange chaotic attractor. In the low coupling regime it is the particle evolving on the periodic attractor that has most influence with respect to the system dynamics. Here a directed current can be seen. However, with increasing strength of coupling both attractors play a key role in the overall dynamics. Indeed, it has been demonstrated that with increasing coupling strength the attractors can merge thus allowing trajectories to explore wider regions of phase-space. We have also shown that the chaos-hyperchaos transition, appearing at a critical coupling strength, marks the point at which a directed current ceases to exist. the frequency and amplitude of the In addition these windows are quite often too narrow to allow for a We performed further studies combining the parameter values from different windows 
of regular and chaotic behaviour occurring in the bifurcation diagrams of the single (uncoupled) system [21]. In general it turns out that a merging crisis is always a precursor to a transition to hyperchaos on the route to vanishing current.

Relating these results to a previous study [15] where both particles evolve (individually) on strange attractors, we find that the system under investigation in this paper promotes directed transport in a regime of much weaker coupling. However, increased coupling can produce contrasting results for both systems. For the system de- scribed above, an increased coupling yields global chaos resulting in zero current, while in [15] a directed current is only possible with an increased coupling such that the chaos is suppressed.

\section{ACKNOWLEDGMENTS}

Numerical computations were done on the Sciama High Performance Compute (HPC) cluster which is supported
[1] W. Acevedo, and T. Dittrich, Prog. Theor. Phys. Suppl. 150, 313 (2003).

[2] H. Schanz, and M. Prusty, Phys. A: Math. Gen. 38, 10085 (2005).

[3] M. Horvat,and T. Prosen, J. Phys. A: Math. Gen. 37, 3133 (2004).

[4] P. Reimann, Phys. Rep. 361, 57 (2002).

[5] D. Hennig, A.D. Burbanks, A.H. Osbaldestin, and C. Mulhern J. Phys. A: Math. Theor. 43, 345101 (2010).

[6] D. Hennig, A.D. Burbanks, and A.H. Osbaldestin, Physica $D$ 238, 2273 (2009).

[7] J.B. Majer et al. Phys. Rev. Lett. 90, 056802 (2003).

[8] R.D. Austumian, and P. Hänggi, Phys. Today. 55, 33 (2002).

[9] P. Hänggi, and F. Marchesoni Rev. Mod. Phys. 81, 387 (2009).

[10] C. Beck Physica C 473, 21 (2012).

[11] S. Fugmann, D. Hennig, L. Schimansky-Geier, and I.M. Sokolov, Eur. Phys. J. Special Topics 191, 187-210 (2010).

[12] A. Smerzi, S. Fantoni, S. Giovanazzi, and S.R. Shenoy Phys. Rev. Lett. 79, 4950-4953 (1997).

[13] E. Ott, Chaos in Dynamical Systems, New York: Cam- bridge University Press, (1993).

[14] S. Yanchuk, and T. Kapitaniak, Phys. Lett. A 290139 (2001).

[15] D. Hennig., A.D. Burbanks, A.H. Osbaldestin, and C. Mulhern, Chaos 21, 023132 (2011).

[16] J. L. Mateos, Phys. Rev. Lett. 84, 258 (2000).

[17] J. L. Mateos, Physica A 325, 92 (2003).

[18] A. Kenfack, S.M. Sweetman, and A.K. Pattanayak, Phys. Rev. E 75, 056215 (2007).

[19] T.S. Parker, and L.O. Chua, Practical Numerical Algorithms for Chaotic Systems, Springer-Verlag (1989).

[20] O. Yevtushenko, S. Flach, and K. Richter, Phys. Rev. E 61, 7215 (2000); S. Denisov, S. Flach. A.A. Ovchinnikov, O. Yevtushenko, and Y. Zolotaryuk, Phys. Rev. E 66, 041104 (2002).

[21] D. Hennig, L. Schimansky-Geier, and P. Hänggi, Eur. Phys. J. B 62, 493 (2008); Europhysics News 39, 21 (2008).

[22] C. Grebogi, E. Ott, and J. A. Yorke, Phys. Rev. Lett., 481507 (1982); J. A. Yorke, Physica D 7, 181 (1983); F. Romeiras and J. A. Yorke, Phys. Rev. A 36, 5365 (1987). 

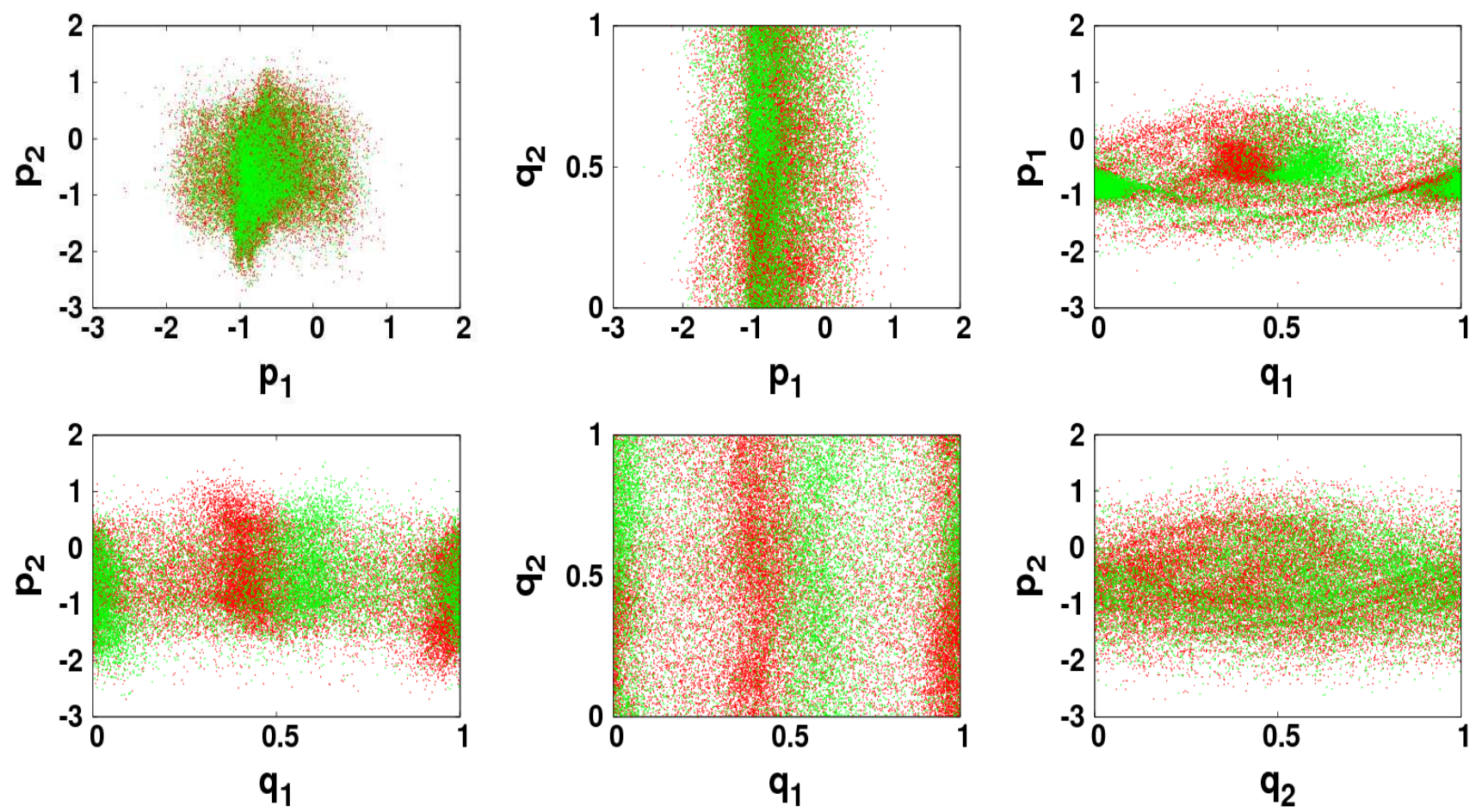

Figure 5. All possible two-dimensional projections (excluding reflections) showing the merging of the two attractors for the case $\kappa=0.4$. The remaining parameter values are given in Fig. 1. 